\title{
Complete surgical myocardial revascularization: shift of paradigm of the gold standard in the current era
}

\author{
Venkat R. Machiraju \\ Department of Thoracic surgery, University of Pittsburgh Medical center, Pittsburgh, PA 15238, USA.
}

Correspondence to: Prof. Venkat R. Machiraju, Department of Thoracic surgery, University of Pittsburgh Medical center, Machiraju, 534 Squaw run road east, Pittsburgh, PA 15238, USA. E-mail: vrmachiraju@aol.com

\begin{abstract}
How to cite this article: Machiraju VR. Complete surgical myocardial revascularization: shift of paradigm of the gold standard in the current era. Vessel Plus 2019;3:25. http://dx.doi.org/10.20517/2574-1209.2019.008
\end{abstract}

Received: 12 Apr 2019 First Decision: 8 May 2019 Revised: 14 May 2019 Accepted: 10 Jul 2019 Published: 20 Jul 2019

Science Editor: Mario F. L. Gaudino Copy Editor: Jia-Jia Meng Production Editor: Jing Yu

\begin{abstract}
Coronary artery bypass surgery is recommended for the patients with symptomatic coronary artery disease or patients having critical left main trunk stenosis. From the inception of the procedure it has undergone several modifications during different time periods evolving into the safe, durable and effective procedure that is to-day. Complete myocardial revascularization (CR) restores blood supply to all the territories in the myocardium that are ischemic because of narrowed blood vessels. Earlier clinical studies from the1980s showed that patients who had CR had better quality of life free of angina with less major adverse coronary events and had survival benefit when followed at 5 years and 10 years compared to the patients who had incomplete myocardial revascularization. According to the coronary artery surgery study registry the benefit is more pronounced when patients had severe triple vessel disease, class III-IV angina and decreased ejection fraction.
\end{abstract}

Keywords: Coronary artery disease, complete myocardial revascularization, off-pump surgery, on-pump surgery, percutaneous coronary intervention

\section{INTRODUCTION}

Both medical and surgical therapeutic interventions of coronary artery disease (CAD) are extensively studied by the patients, physicians, hospital administrators, epidemiologists, and the insurance companies because of the risks involved to the patient on one side and the cost of care on the other side. CAD still 
remains as one of the major healthcare entities that drains Billions of dollars and the treatment at best is only a temporizing situation without a permanent cure for the disease entity. The patient's genetic background, dietary habits, stress, lack of exercise, diabetes, smoking, other socioeconomic conditions and many more factors contribute towards varying outcomes. As physicians became comfortable with the existing methods of treatments, newer drugs and surgical techniques, percutaneous catheters and varying stents came into the field that have provided the impetus for physicians to constantly learn while providing care. This has resulted in improvements on one side and ongoing sporadic catastrophic events on the other $\operatorname{side}^{[1,2]}$. This paper will outline the changes that have occurred over the years on the overall treatment of the disease.

\section{EVOLUTION OF THERAPEUTIC OPTIONS}

It was believed that the occlusion of coronary arteries led to myocardial ischemia or infarction from the pathological specimens. But it was not until Sones and Shirely ${ }^{[3]}$ performed several hundred coronary cine angiograms and showed the lesions in the coronary artery branches and their anatomical variations, surgeons could not plan a method of treatment to tackle the occlusions. Several advances occurred simultaneously that incorporated cardiopulmonary bypass routinely into cardiac surgery and surgeons gained experience to tackle peripheral vascular disease as well. Development of microvascular instruments, fine suture materials, wearing surgical loupes and a head light for better visualization have helped to improve technical proficiency for coronary artery bypass surgery (CABG). Improvements in myocardial protection starting from intermittent aortic cross clamping and a fibrillating heart to a completely quiescent heart with antegrade and retrograde blood cardioplegia gave an enormous leap forward for CABG surgery.

Saphenous vein was the preferred conduit until late 1970s in CABG surgery. At that time, it was shown to have a better patency when used to bypass left (Lt) anterior descending (LAD) artery, though there are graft failures to the other branches of coronary arteries. LAD being the most important blood vessel, was bypassed with the best quality of the available vein. The graft being straight and short, carried high blood flow into the large myocardial segment. These factors contributed to the high patency rate. A vein which lies normally in a low-pressure vascular system, once subjected to arterial pressure and blood flow, showed evidence of complete endothelial cell damage of the intima and subsequent development of rapid atherosclerosis. Though use of Lt internal thoracic artery (LITA/LIMA) in CABG was performed much earlier by Kolesov, universal acceptance by surgeons did not happen until Floyd Loop's ${ }^{[4]}$ landmark article about the long-term benefits of arterial graft to LAD using LITA. Subsequently as more and more data became available about vein graft atherosclerosis, surgeons have slowly adopted the use of more arterial grafts using right internal thoracic, radial (RA) and gastro epiploic arteries.

\section{COMPLETE SURGICAL MYOCARDIAL REVASCULARIZATION}

Before percutaneous coronary intervention (PCI) became a generally acceptable procedure to treat coronary artery stenosis, surgical revascularization with bypass grafts had been the standard of care to treat patients with significant CAD. During the peak of surgical myocardial revascularization in late 80s, CABG was only performed in large Medical centers in the hands of highly trained surgeons who were able to tackle even small blood vessels the size of $1 \mathrm{~mm}$. Anatomically right (Rt.) Coronary artery has a rt. ventricular branch, posterior descending (PDA) and posterolateral (PLB). Lt coronary artery has LAD, 1 or 2 diagonal branches and main circumflex (CX) branch giving several marginal branches on the posterior wall of the Lt. ventricle. Between the LAD and CX a large ramus branch can come on to the lateral wall. Every suitable vessel with 70\% or above stenosis was bypassed. Surgeons were able to perform 3 to 6 bypasses on an average and even the number increased to 7 or 8 depending on the coronary anatomy and diffuse nature of the disease. Complete surgical revascularization is accomplished when all the stenotic blood vessels are bypassed. While this is considered as an anatomical classification, a functional 
classification is used when the blood vessels in the ischemic zones of the myocardium are only bypassed ignoring the areas that already had myocardial infarction and myocardial scarring. Number of distal anastomosis are counted and matched to the number of stenosis in the blood vessels that are technically by passable todetermine whether a CR or incomplete myocardial revascularization (IR) is performed.

In anatomical classification, a completely occluded blood vessel proximally is bypassed as long as it is open distally and is suitable for bypass. It is considered that next to the scar tissue there is a zone of ischemic myocardium that can get benefit from blood flow through the bypass graft. It can also provide retrograde collateral flow to the other connecting vessels. The typical example is bypassing an open distal Rt. Coronary artery after it's complete proximal occlusion. Most of the patients had at-least one arterial graft to $\mathrm{LAD}$ and the remaining grafts were performed with saphenous vein. LAD revascularization is the key part of CR when-ever there is evidence of $70 \%$ or more stenosis.

LAD atherosclerosis presents in different forms: (1) Proximal severe stenosis with excellent distal vessel and a bypass graft can be sutured anywhere distal to the stenosis; (2) Proximal stenosis followed by an open LAD along with diffuse atherosclerosis in the middle and the distal LAD is open at the apex of the Lt ventricle requiring bypass to both proximal and distal segments to protect the entire septum as well the anterior and apical segments of the myocardium; and (3) completely occluded LAD with atherosclerotic process and will need extensive endarterectomy to revascularize the vessel. Such large endarterectomy not only removes the atherosclerotic plaque from the LAD but also removes the plaque from the opening of the septal branches and can improve the blood flow to the interventricular septum. By-passes requiring to diagonal, ramus and marginal branches on the Lt side will add up to 7 or 8 distal anastomosis very easily. Apart from LAD, bypassing another major vessel in the Lt coronary system has also increased the survival benefit.

Use of all arterial grafts to the Lt coronary system was popularized by creating a LIMA+RIMA as a "T" graft and performing multiple sequential grafts to the marginal branches with free RIMA graft and LIMA being anastomosed to the LAD as an in situ graft. Prior to "T" grafting became popular, my preferred operation was an in situ RIMA graft across the midline to LAD and LIMA graft to an OM branch whenever applicable. In aortic arch disease there is higher chance of Lt subclavian artery to get occluded than innominate artery. The next arterial graft that got popularized is the RA graft taken from the nondominant hand. This is also used as a free graft from the aorta or as " $\mathrm{T}$ " graft from the LIMA graft ${ }^{[5-7]}$.

The patients that were discharged from the hospital with-out any perioperative morbidity had performed well, free of anginal symptoms and without repeat hospital readmissions for myocardial infarction or heart failure. Zimarino et al ${ }^{[8]}$ reviewed 28 studies that included 8,3695 patients that were treated with surgery as well as PCI. Patients who had CR with multi vessel coronary artery disease (MVCAD), at 4.5 years follow up showed less mortality and less repeat further interventions. CR performed as an elective surgical procedure has better outcomes over medical therapy or PCI in diabetic patients with MVCAD. Takagi et al. ${ }^{[9]}$ did metanalysis of patients from fourteen studies and compared 3,0389 patients and found that patients who had complete surgical revascularization did have 37\% less mortality when followed over a period of time compared to similar group of patients that had IR.

\section{REASONS FOR SHIFT OF PARADIGM}

Changes in the trends of bypass grafting using multiple arterial grafts, introduction of "off-pump" CABG and operating on several coronary artery branches with multiple stents and advances in PCI procedures, complicated the CABG. Emergency surgical revascularization on patients who are loaded with antiplatelet agents also precluded prolonged surgical procedures for fear of perioperative bleeding complications. Collaborative effort between cardiologists and cardiac surgeons to address CAD, led to hybrid procedures 
where a durable LITA graft to LAD is created through a Lt anterior small thoracotomy ${ }^{[10]}$ and remaining stenotic vessels were dilated and stented by the cardiologists. In addition, the mean age of patients having surgery became much higher and several comorbidities that increased overall surgical risk. Pre-existing cerebrovascular disease, chronic hemodialysis, advanced malignancy and severe chronic obstructive lung disease are few conditions to name that influenced plan of surgical treatment. More females are having surgery who are in general are higher surgical risk compared to men with similar risk factors. The difficulties encountered in constructing a satisfactory bypass graft through a small thoracotomy incision resulted in off-pump bypass surgery through median sternotomy. This approach also broadened the access to bypass other vessels like Rt. and Cx. coronary artery branches.

With rapid expansion of cardiac surgery into the community hospital setting, the total number of CABG surgeries performed by an individual surgeon has significantly decreased and simultaneously the experience to tackle difficult coronary surgical cases. They have adopted the general notion that bypassing one vessel in each territory of myocardium is enough to relieve the patient of his anginal symptoms and multiple bypass grafts will only prolong the operation and subsequently increase the immediate post op complications like perioperative bleeding, low cardiac output syndrome and need for prolonged ventilatory support. At one time majority of the surgeons had the comfort zone and experience to expose deep intramuscular LAD embedded in the interventricular septum, perform extensive endarterectomies in the vessels with diffuse atherosclerosis and bypass even the main CX coronary artery in the atrioventricular (AV) groove when the marginal branches were too many and too small for bypass. Generally main CX in the AV groove is free of atherosclerosis and is of larger caliber. Though coronary vessels are epicardial, some-times the proximal LAD and ramus or the first marginal branches are deeply embedded in the cardiac muscle that needed patience to identify and dissect out these vessels for bypass. These intramuscular vessels are again free of atherosclerosis that they are best suitable for bypass. These anatomical variations are the cause of IR in the hands of less experienced Surgeons. The difficulty is compounded in obese patients where the heart is covered with lot of fatty tissue and during redo coronary artery bypass graft surgery. PCI also became an established modality to treat CAD and experienced interventional cardiologist had shown excellent results in stenting multiple stenotic coronary arteries. Vein graft atherosclerosis resulted in stenotic bypass grafts which required redo-CABG. In late 90 s, $15 \%-20 \%$ of the coronary artery surgical volume in all major surgical centers happened to be redo bypass surgery. With the advances in medical therapy and PCI, redo bypass surgery has significantly decreased. Perioperative use of statins helped to stabilize vein graft atherosclerosis so that cardiologists are able to address vein graft stenosis. As the surgical volume decreased, the peripheral cardiac surgical centers which at one time had two cardiac surgeons, are able to hold on to only one cardiac surgeon who doesn't want to take excessive responsibility of tackling difficulty cases. Public awareness of individual surgeon's data including surgical volume, mortality, morbidity and hospital readmissions following CABG surgery by the local state governments did not help the matters either. The number of grafts they are performing had come down as evidenced by the society of thoracic surgeons (STS) data base published in 2018. As per the STS the published data in 2016 showed that in spite of increase in CABG procedures per the year by $6.1 \%$ to a total of 156,931, the number of 4 and 5 distal anastomosis are much fewer than the previous years ${ }^{[11]}$. As the isolated CABG procedures have gone down there has been a significant increase of valve with CABG procedures like aortic valve replacement or mitral valve/replacement. This has changed the complexity of the surgery as such surgeons focused on the main coronary artery branches for revascularization and proceeded with valve surgery. This is evidenced by the higher pre-operative risk assessment both by euroscore and STS risk calculator.

\section{Off-pump coronary artery bypass surgery}

While majority of cardiac surgeons are able to perform a satisfactory bypass to the blood vessels that are in the front of the heart while the heart is beating, only a few surgeons could master the technique 
of bypassing all the blood vessels both in the back and on the lateral wall of the heart with-out much hemodynamic compromise. Avoiding the heart lung machine eliminated the complications related to it, and also decreased the cost of the procedure which in turn contributed to the exponential growth of CABG surgery in the third world countries. In the hands of less skilled surgeons the procedure instead of becoming a "beating heart surgery" became a "beat the heart" surgery. Intra operative conversion from off-pump to on-pump surgery showed increased perioperative morbidity and mortality. Off-pump surgery became an accepted approach when the ascending aorta appeared calcified and the chances of atherosclerotic emboli to the brain from the aorta are considered high ${ }^{[7]}$. Benedetto et al ${ }^{[12]}$ reviewed CABG patients that had surgery at Bristol Heart Institute, in England both off-pump 7427 pts and on-pump 7128 pts and showed that patients who had multiple bypass grafts lived longer when compared to the patients that had fewer number of grafts. Afilalo et al. ${ }^{[13]}$ published meta-analysis and meta-regression of 8961 patients and found that there is $30 \%$ stroke reduction in off-pump coronary artery bypass while mortality and myocardial infarction rates are the same in both groups. The decrease in stroke rate is attributed to avoidance of aortic manipulation. The benefit is apparent when aorta is not at all touched, but if the aorta is partially occluded to perform proximal anastomosis the risk of stroke is reintroduced whether the procedure performed is on-pump or off-pump. Because of the improvements in myocardial protection, now most procedures are performed on cardiopulmonary bypass with single aortic cross clamping.

The ROOBY trial studied 2203 patients randomized to either off or on pump CABG from 2002-2007. The 5 -year mortality was $11.9 \%$ in on-pump $v$ s. $15.2 \%$ in the off-pump group ${ }^{[14]}$. The on-pump patients required fewer coronary interventions in the subsequent years. Now off-pump CABG is falling out of favor for triple vessel disease and can still be used for isolated one or two vessel disease involving anterior targets.

Minimally invasive procedures such as mini-thoracotomy along with endoscopic and robot assisted CABG procedures evolved but could not gain popularity as the cost and time involved to learn the required minimal skills is high and protracted.

\section{SURGERY VS. PCI}

In randomized studies comparing PCI vs. surgery, patients who underwent complete revascularization performed better when compared to patients with incomplete revascularization. When PCI is guided by the fractional flow reserve (FFR) evaluation, there may be fewer number of stent placements when compared to the angiographic observation of stenosis in the coronary arteries. It is still considered as $\mathrm{CR}$ as there is substantial evidence that FFR above 0.8 can be treated medically and does not need therapeutic intervention ${ }^{[15]}$. Tackling the culprit vessel fared well when patient came to the hospital with acute ST segment elevation myocardial infarction. While some cardiologists performed multiple vessel PCI at the same time, majority preferred multiple staged procedures requiring repeated interventions and hospital admissions. In arterial revascularization therapy study 1143 patients that were randomized for either surgery or angioplasty, the patients that had complete revascularization performed better when compared to the patient that had incomplete revascularization with angioplasty ${ }^{[16]}$.

\section{BYPASS CONDUITS}

Saphenous vein quality is influenced by patient's age, sex, past history of phlebitis, pregnancy, and occupation. The technique of saphenous vein harvest also undergone several surgical modifications starting from one large skin incision from groin to the ankle to multiple skip incisions and finally endoscopic removal. There is no uniform standard for vein preservation solution. Perioperative use of antiplatelet agents and statin therapy have increased the vein graft patency as well the longevity. Endoscopic removal of the saphenous vein has become the standard practice for both cosmetic reasons as well as avoiding wound healing complications in the leg with open surgical removal. Saphenous vein is more adoptable and 
much easier to use for multiple sequential grafting than mammary arteries or RA artery. LIMA graft has varying size and blood flow depending upon the body size and patient's age. Lt subclavian artery stenosis used to be a problem in the pre-angioplasty era but now even a critical stenosis in the subclavian artery is successfully dilated and stented, restoring blood flow in the mammary graft. Initially the mammary artery was removed from underneath sternum as a pedicle graft but skeletonizing the internal mammary became the preferred method, leaving capillary collaterals behind to feed the sternal edges. This technique is highly recommended in diabetics, females and whenever bilateral mammary arteries are used for bypass surgery. However skeletonizing the artery had challenges like injury to the arterial wall, development of intramural hematoma and intimal dissection leading to failure to use the graft. Various vasodilator pharmacological agents can be instilled over the mammary pedicle in-order to dilate the vessel and increase the blood flow. While the LIMA graft is used preferentially as an in situ graft, RIMA is used as an in situ or as a free graft depending upon the planned surgery. RA artery became the alternate arterial conduit after mammary arteries but developed arterial spasm because of the thick muscular wall and also evidence of calcification in elderly patients. Perioperative use of Calcium channel blockers has increased the patency of the RA artery graft but the limitation is that it's use is recommended to bypass the vessels with $90 \%$ or more stenosis to avoid any competitive blood flow. It showed better patency on the Lt coronary artery system than on the right side. Though RA artery showed better long-term patency as compared to the saphenous vein graft, because of the limitations it had, it is not as frequently used as recommended in the literature. The use of RIMA as a second arterial graft to the Lt coronary system obviated the need for a third arterial graft. Removal of both RIMA and RA artery became a time-consuming operation with added complications, and surgeons didn't want to use both unless there is definite benefit to the patient. The gastroepiploic artery also is prone for spasm and showed inconsistent blood flow. Constant research is going to improve the patency of the vein grafts by external stenting or other methods or to find a synthetic graft that can be used when patient doesn't have suitable conduit.

There was a push by Taggart in UK, Puskas in $\mathrm{USA}^{[7]}$ for performing CABG with only arterial grafts. Using both internal thoracic arteries for CABG surgery was promoted as a must to do thing. It did not become popular among American surgeons as majority of the patients that came for surgery are not only old but have several comorbidities that increased their over-all risk. After following for 10 years other than a LIMA graft to the LAD using all arterial grafts when compared to a single arterial graft and remaining venous grafts, didn't increase the survival benefit in elderly patients but the younger patients may benefit from multiple arterial grafts if their natural life expectancy is 10 years or beyond. The survival benefit of any procedure depends upon the biological and anatomical age of the patient. That is also true with CR as younger patients tend to live longer and symptom free while older patients with low ejection fraction tend to have heart failure symptoms ${ }^{[17]}$.

\section{CONCLUSION}

As the saying goes "There was never a successful incomplete operation". The gold standard of CABG still remains as the CR performed by an experienced operator so that all the blood vessels with critical stenosis can be tackled. The goal can be achieved by off-pump or on-pump surgery or by PCI. Complete revascularization rewards patients with symptom free or risk free postprocedural period. There were several clinical trials that studied CAD and therapeutic interventions over the years. There may be minor differences in the conclusions but majority of the studies do concur that CR gives better survival and symptom free life.

\section{SPECIAL NOTE}

This article reflects the surgical practice that is popular in USA and may vary from the common practice globally. 


\section{DECLARATIONS}

\section{Authors' contributions}

Writing-original draft preparation and editing: Machiraju VR

\section{Availability of data and materials}

Not applicable.

\section{Financial support and sponsorship}

None.

\section{Conflicts of interest}

The author declared that there are no conflicts of interest.

\section{Ethical approval and consent to participate}

Not applicable.

\section{Consent for publication}

Not applicable.

\section{Copyright}

(c) The Author(s) 2019.

\section{REFERENCES}

1. Jones EL, Craver JM, Guyton RA, Bone DK, Hatcher CR, et al. Importance of complete revascularization in performance of the coronary bypass operation. Am J Cardiology 1983;51:7-12.

2. Bell MR, Gersh BJ, Schaff HV, et al. Effect of completeness of revascularization on long-term outcome of patients with three-vessel disease undergoing coronary artery Bypass surgery. A report from the Coronary Artery Surgery Study (CASS) Registry. Circulation 1992;86:446-57.

3. Loop FD. Coronary artery surgery. Ann Thorac Surg 2005;79:S2221-7.

4. Loop FD, Lytle BW, Cosgrove DM, Stewart RW, Goormastic M, et al. Influence of the internal-mammary-artery graft on 10-year survival and other cardiac events. N Engl J Med 1986;314:1-6.

5. Tatoulis J. Total arterial revascularization-patient selection, stenosis, conduits, targets. Ann Cardiothorac Surg 2013;2:499-506.

6. Taggart DP, Benedetto U, Gerry S, Altman DG, Gray AM, et al. Bilateral versus Single Internal-Thoracic-Artery grafts at 10 years. N Engl J Med 2019;380:437-46.

7. Puskas J, Yangawa B, Taggart D. Off-pump, multiple arterial grafting with minimal aortic manipulation: Is it for everyone? J Thorac Cardiovasc Surg 2016;151:4-6.

8. Zimarino M, Ricci F, Romanello M, Di Nicola M, Corazzini A, et al. Complete myocardial revascularization confers a larger clinical benefit when performed with the state-of-the-art techniques in high risk patients with multivessel coronary artery disease. A metaanalysis of randomized and observational studies. Cathet Cardiovasc Interv 2016;87:3-12.

9. Takagi H, Wantanabe T, Mizuno Y, Kawai N, Umemoto T. A meta -analysis of adjusted risk estimates for survival from observational studies of complete versus incomplete revascularization in patients with multivessel disease undergoing coronary artery bypass grafting. Interact Cardiovasc Thorac Surg 2014;18:679-82.

10. Calafiore AM, Giammarco GD, Teodori G, Bosco G, D’Annunzio E, et al. Left anterior descending coronary artery grafting via left small Thoracotomy without cardiopulmonary Bypass. Ann Thorac Surg 1996;61:1658-63.

11. D’Agostino RS, Jacobs JP, Badhwar V, Fernandez FG, Paone G, et al. The Society of Thoracic Surgeons Adult Cardiac Surgery Database: 2018 Update on Outcomes and Quality. Ann Thorac Surg 2018;105:15-23.

12. Benedetto U, Caputo M, Patel NN, Fiorentino F, Bryan A, et al. Long- term survival after Off-Pump versus on-pump coronary artery bypass Surgery. Does completeness of revascularization play a role? Int J Cardiol 2017;246:32-6.

13. Afilalo J, Rasti M, Ohayon SM, Shimony A, Eisenberg MJ. Off-pump vs. on-pump coronary artery bypass surgery: an updated metaanalysis and meta-regression of randomized trials. Eur Heart J 2012;33:1257-67.

14. Shroyer AL, Hattler B, Wagner TH, Collins JF, Baltz JH, et al. Five-Year Outcomes after On-Pump and Off-Pump Coronary -Artery bypass. N Engl J Med 2017;377:623-32.

15. van den Brand MJ, Rensing BJ, Morel MA, Foley DP, de Valk V, et al. The effect of completeness of revascularization on event-free survival at one year in the ARTS trial. J AM Coll Cardiol 2002;39:559-64. 
16. Girerd N, Magne J, Rabilloud M, Charbonneau E, Mohamadi S, et al. The impact of complete revascularization on long-term survival is strongly dependent on age. Ann Thorac Surg 2012;94:1166-72.

17. Nicolas Girerd, Julian Magne, Muriel Rabilloud, Eric Charbonneau et al. The Impact of complete revascularization on Long-Term survival is strongly dependent on age. Ann Thorac Surg 2012;94:1166-72. 